\title{
Teneurs en acides gras polyinsaturés essentiels du lait maternel en France : évolution du contenu en acides linoléique et alphalinolénique au cours des $\mathbf{1 0}$ dernières années
}

\author{
Carole BOUÉ-VAYSSE ${ }^{1}$ \\ Claude BILLEAUD ${ }^{2}$ \\ Philippe GUESNET ${ }^{3}$ \\ Leslie COUËDELO ${ }^{1}$ \\ Jean-Marc ALESSANDRI ${ }^{3}$ \\ Guy PUTET ${ }^{4}$ \\ Nicole $\mathrm{COMBE}^{1}$ \\ 1 ITERG, Nutrition Métabolisme \& Santé,
Université Bordeaux 1, \\ Avenue des Facultés, 33405 Talence Cedex \\ $<$ n.combe@iterg.com> \\ ${ }^{2}$ CEDRE, Lactarium, 33000 Bordeaux \\ ${ }^{3} \mathrm{Nu}$. Re.Li.Ce, UR909, INRA, \\ F 78352-Jouy-en-Josas \\ ${ }^{4}$ Association des Lactarium de France, \\ 69000 Lyon
}

\begin{abstract}
Background: Long-chain polyunsaturated fatty acids (LC-PUFA) are nutritionally important constituents of breast milk to support normal growth, immune function and central nervous system development of newborn infants. Both linoleic acid (18:2 n-6; LA) and alpha-linolenic acid (18:3 n-3 ; $A L A)$, the essential fatty acids, precursors of $n-6$ and $n-3$ LC-PUFA, are also present in breast milk. Although the total amount of fat in human milk is fairly constant, the fatty acid composition can vary substantially depending on the diet of the mother. Large variations in the LA, ALA and DHA (22:6n-3) contents are observed among countries. As reported by previous studies (1993-2001), the ALA consumption of the French population was 2-3 times lower than the recommended value. Since this period, the food industry offers more food products enriched in n-3 fatty acids. Objective: The present study aimed to investigate whether current recommendations and improved n-3 PUFA content of food products comply with the actual breast-milk PUFA composition. For this purpose, the PUFA content of a large number of human milk samples collected in 2007 in different French regions were compared with previous data obtained in studies conducted in the nineties years (1993-1998). Moreover, this study aimed to confirm expected reduction of the trans fatty acid (TFA) intake resulting from decreased level of TFA in margarine and other dietary fats during the same period. Study design and methods: Mature (postpartum day $\geq 30$ ) human milk samples $(n=145)$ were provided in 2007 by eight French regional human milk banks (Bordeaux : $n=20$; Dijon: $n=21$; lle-de-France: $n=18$; Lyon: $n=20$; Montpellier: $n=18$; Nantes: $n=21$; St Etienne: $n=6$; Tours: $n=21$ ), from healthy women volunteers with no particular health history. The fatty acid composition of breast milk was analysed by high-resolution gas-liquid chromatography. Statistical analysis was performed with Kruskall Wallis and Mann and Whitney $U$ tests $(p<0.05)$. Results: Fatty acid composition of human milk samples did not differ significantly among the eight French regions. The proportions of saturated fatty acids were relatively similar (means ranging from $44.9 \%$ to $48.7 \%$ of total fatty acids). Monounsaturated fatty acids also varied little, from $36.9 \%$ to $39.7 \%$. Total $n-6$ and $n-3$ PUFA accounted for $12.5 \%-15.2 \%$, with an $n-6 / n-3$ PUFA ratio close to 10, similar among the collect centers. Considering the overall results, LA represented between $10.4 \%$ and $12.7 \%$, and ALA varied from $0.72 \%$ to $0.95 \%$. Comparison of our results to previous data (1993-1998) showed opposite changes for LA and ALA. During the last decade, the LA level has decreased by $17 \%$, whereas the ALA level has increased by $45 \%$, and hence in 2007 , the LA/ ALA ratio was close to 14 versus 24 in 1993-1998. The LC-PUFA content remained unchanged. Concerning total TFA, their content was lower $(-37 \% ; p<0.0001)$ in 2007 than in 1997, respectively 1.3\% and $2.1 \%$ of total fatty acids of breast milk. Conclusion: Basing on the PUFA composition of human milk, this large French study shows favourable changes in consumption of both omega- 6 and omega- 3 fatty acid precursors, and in TFA, in the course of last decade.
\end{abstract}

Key words: human milk, essential fatty acids, long-chain polyunsaturated fatty acids, trans fatty acids

nique (20:4 n-6;ARA) et docosahexaénoïque $(22: 6 n-3 ; D H A)$, ainsi que leurs précurseurs métaboliques respectifs, les acides linoléique (18:2 n-6; LA) et alphalinolénique (18:3 n-3; ALA) $[1,2]$. Pour couvrir ses besoins en ARA et DHA, les deux AGPI longues chaînes (AGPI-LC) majoritaires du cerveau, dont les contenus augmentent jusqu'à l'âge de 2 ans, le nourrisson utilise les AGPI-LC présents dans le lait ainsi que ceux qu'il synthétise à partir des précurseurs LA et ALA. Cependant, chez les enfants prématurés mais également chez les enfants nés à terme, les quantités synthétisées, en particulier celles de DHA, apparaissent insuffisantes en regard des besoins. Sur le plan du développement neurosensoriel, le bénéfice d'une supplémentation en DHA a été clairement démontré $[3,4]$. Les résultats semblent cepen- 
dant moins consensuels lorsqu'il s'agit d'enfants nés à terme [5].

Si le lait humain contient une quantité de lipides relativement constante, sa composition en acides gras peut varier de façon substantielle en fonction de l'alimentation de la mère $[6,7]$. Des études d'observation et d'intervention ont montré que les niveaux de LA, ALA, DHA ainsi que ceux de l'acide oléique et des acides gras trans (AGT) mesurés dans le lait humain variaient avec les niveaux de ces acides gras dans I'alimentation maternelle, soulignant ainsi l'intérêt de ce marqueur biologique (pour plus d'informations, se reporter à la revue de S. Innis [8]). Les données de consommation d'AGPI $n-6$ et $n-3$ les plus récentes pour des femmes françaises concernent les années 1997-99 [9]. Elles ont montré que l'apport recommandé en ALA n'était pas atteint $(0,8 \mathrm{~g} / \mathrm{j}$ vs $2 \mathrm{~g} / \mathrm{j})$, entraînant un déséquilibre du rapport oméga-6/oméga-3 dont la valeur se situait en moyenne à 14-15 contre 5 pour la valeur recommandée. Ce faible niveau de consommation en ALA a été également retrouvé dans le lait maternel prélevé à cette même période [10-15].

En 2003, I'AFSSA a produit un rapport sur l'intérêt nutritionnel des acides gras oméga- 3 dans le risque cardiovasculaire, incitant à accroître les apports en précurseurs et/ou longues chaînes oméga-3 [16]. Un plus grand éventail de produits "sources ou enrichis en oméga-3 est offert aux consommateurs depuis ces dernières années. Dans ce contexte, l'objectif de l'étude présente était d'apprécier l'évolution des consommations d'AGPI n- 6 et n- 3 via la comparaison de leurs contenus dans des échantillons de lait humain mature collectés en 2007 dans 8 régions réparties sur le territoire français et des échantillons recueillis lors d'études conduites entre 1993 et 1998. Cette étude a permis également d'évaluer la baisse attendue de la consommation d'AGT par la population française, suite à la diminution des teneurs en AGT des produits alimentaires au cours des 10 dernières années. Des données récentes collectées par I'Institut Français pour la Nutrition (IFN) indiquent que la teneur en AGT des margarines et de la majorité des produits transformés a fortement diminué, pour représenter en moyenne aujourd'hui moins de 0,5 g d'AGT pour $100 \mathrm{~g}$ de produit fini [17].

\section{Matériel et méthodes}

L'étude menée entre juillet et décembre 2007 a porté sur 145 échantillons de lait maternel mature (> 30 j d'allaitement) collectés dans 8 lactariums français (Bordeaux : $\mathrm{n}=20$; Dijon : $\mathrm{n}=21$; île-de-France $: \mathrm{n}=18$; Lyon $: \mathrm{n}=20$; Montpellier: $\mathrm{n}=18$; Nantes: $\mathrm{n}=21$; St-Étienne $: n=6$; Tours $: n=21$ ). Les partici- pantes de l'étude ont été informées des objectifs avant recueil de leur consentement écrit. Les échantillons de lait $(20 \mathrm{~mL}$ prélevés sur l'expression totale d'un sein, lors du $1^{\text {er }}$ allaitement de la journée) ont été rapidement congelés et stockés à - $20^{\circ} \mathrm{C}$ jusqu'à l'analyse.

La composition en acides gras des échantillons de lait maternel a été déterminée par chromatographie en phase gazeuse (CPG) haute performance après préparation des esters méthyliques totaux par la méthode de Lepage et Roy [18]. Brièvement, $100 \mu \mathrm{L}$ de lait maternel ont été mis en présence de $2 \mathrm{~mL}$ d'un mélange méthanol-isooctane $(4 / 1 ; \mathrm{v} / \mathrm{v})$ et de $200 \mu \mathrm{L}$ de chlorure d'acéthyle. Les esters méthyliques d'acides gras (EMAG) ainsi obtenus ont été analysés avec un chromatographe (Trace Ultra-Thermo Electron Corp, France) équipé d'un détecteur à ionisation de flamme et $d^{\prime}$ une colonne capillaire en silice greffée (BPX $70,60 \mathrm{~m} \times 0,22 \mathrm{~mm}$ i.d., épaisseur de film : $0,25 \mu \mathrm{m} ; \mathrm{SCE}$, France); le gaz vecteur était I'hélium (pression d'entrée : $100 \mathrm{kPa}$; rapport de fuite 1/60). Le programme de montée en température de la colonne était le suivant : de $150^{\circ} \mathrm{C}$ jusqu'à $200^{\circ} \mathrm{C}$ à raison de $1,5^{\circ} \mathrm{C} / \mathrm{min}$, maintien à $200{ }^{\circ} \mathrm{C}$ pendant 30 minutes puis montée en température de $200^{\circ} \mathrm{C}$ à $230^{\circ} \mathrm{C}$ $\left(10^{\circ} \mathrm{C} / \mathrm{min}\right)$ et maintien à $230^{\circ} \mathrm{C}$ jusqu'à la fin de l'analyse (30 $\mathrm{min})$. Les températures de l'injecteur et du détecteur étaient respectivement de $250^{\circ} \mathrm{C}$ et $280^{\circ} \mathrm{C}$. L'identification des différents EMAG est basée sur la comparaison de leurs temps de rétention avec ceux d'EMAG standards (Sigma), chromatographiés dans les mêmes conditions. La quantité de chaque acide gras est exprimée en pourcentage des acides gras totaux.

La comparaison des données issues des 8 lactariums a été faite par le test statistique non paramétrique de Kruskall et Wallis. Le test $U$ de Mann et Whitney a été appliqué pour comparer les données de 2007 à celles de 1997 (différences significatives au seuil de $P=0,05$ ).

\section{Résultats}

\section{Composition en acides gras des échantillons de lait maternel collectés dans les 8 lactariums français}

Les compositions moyennes et écarts-types en acides gras des 145 échantillons de lait maternel sont donnés dans le tableau 1. Elles sont très homogènes d'un centre de collecte à l'autre. En effet, les acides gras saturés (AGS) constituent entre 44,9\%(St-Étienne) et 48,7 \% (Bordeaux et Île-de-France) des acides gras totaux en fonction du centre de collecte ; ils sont principalement représentés par les acides palmitique (16:0), stéarique (18:0), myristique (14:0) et laurique (12:0). L'acide gras saturé prépondérant est le $16: 0$ (23\% en moyenne), les trois autres catégories d'AGS se situent autour de $7 \%$. Les acides gras mono-insaturés (AGMI) représentent entre 36,9\% (Dijon) et 39,7\% (Lyon) en fonction du centre, l'acide oléique étant majoritaire (32,4\% en moyenne).

Les proportions d'AGPI totaux (n-6 et n-3) varient de $12,5 \%$ à 15,2 \% en fonction du lactarium d'origine, avec un rapport entre $n-6$ et n-3 situé autour de 10, qui est similaire entre les centres. L'acide linoléique (18:2 n-6) est le principal représentant des AGPI n-6 ; son pourcentage varie de 10,4 \% (Bordeaux) à 12,7 \% (StÉtienne). Les proportions d'acide arachidonique (20:4n-6) sont comprises entre 0,36\% et $0,45 \%$. Concernant les AGPI n-3, l'acide alphalinolénique (18:3n-3) en est le représentant majeur (65\% des AGPI n-3) ; ses proportions varient de 0,72\% (Bordeaux, Îlede-France) à 0,95 \% (Lyon) par rapport aux acides gras totaux du lait. Comparativement, le $\mathrm{DHA}(22: 6 \mathrm{n}-3)$ se situe entre 0,19\% (Montpellier) et 0,28-29\% (Nantes, Dijon), le DPA (22:5 n-3) entre $0,11 \%$ et $0,15 \%$. Quant à I'EPA (20:5 n-3), il est un constituant mineur du lait maternel (0,05-0,09\%).

Les pourcentages $\mathrm{d}^{\prime} \mathrm{AGT}$ tous isomères confondus sont similaires entre centres de collecte, compris entre $1,27 \%$ et 1,32 \% par rapport aux acides gras totaux. Ils sont représentés à $74 \%$ par les isomères de position 18:1 trans, $21 \%$ par les isomères de position 18:2 trans et $5 \%$ par l'isomère 16:1 9trans.

Le traitement statistique de ces résultats a indiqué l'absence de différence significative entre les profils d'acides gras des échantillons de lait maternel issus des 8 centres de collecte.

\section{Discussion}

L'étude présente évalue le profil en acides gras de 145 échantillons de lait humain mature collectés en 2007 dans 8 régions réparties sur le territoire français, soit une vingtaine d'échantillons par centre de collecte, à l'exception de StÉtienne $(n=6)$; il s'agit à ce jour de la plus importante étude de ce type conduite en France. Les conditions de recueil et d'analyse des échantillons de lait maternel issus des 8 lactariums étant identiques, leur comparaison en fonction de l'origine a pu être réalisée. II n'est ressorti aucune différence significative entre les profils d'acides gras des 8 régions. Sachant que les contenus en acides gras essentiels n- 6 et n-3 du lait humain sont influencés par la présence de ces acides gras dans I'alimentation maternelle, ces résultats suggèrent que la consommation de ces acides gras est similaire d'une région à l'autre. Pour apprécier l'évolution des contenus en AGPI n-6 et n-3 du lait maternel au cours de la dernière décennie, nous avons com- 
Tableau 1. Composition en acides gras (\% pds) des laits maternels de différentes régions françaises

\begin{tabular}{|c|c|c|c|c|c|c|c|c|c|c|c|c|c|c|c|c|}
\hline & \multicolumn{2}{|c|}{$\begin{array}{l}\text { Montpellier } \\
(n=18)\end{array}$} & \multicolumn{2}{|c|}{$\begin{array}{l}\text { IDF } \\
(n=18)\end{array}$} & \multicolumn{2}{|c|}{$\begin{array}{l}\text { St-Étienne } \\
(n=6)\end{array}$} & \multicolumn{2}{|c|}{$\begin{array}{l}\text { Nantes } \\
(n=21)\end{array}$} & \multicolumn{2}{|c|}{$\begin{array}{l}\text { Dijon } \\
(n=21)\end{array}$} & \multicolumn{2}{|c|}{$\begin{array}{l}\text { Tours } \\
(n=21)\end{array}$} & \multicolumn{2}{|c|}{$\begin{array}{l}\text { Bordeaux } \\
(n=20)\end{array}$} & \multicolumn{2}{|c|}{$\begin{array}{l}\text { Lyon } \\
(n=20)\end{array}$} \\
\hline & Moy & ET & Moy & ET & Moy & ET & Moy & ET & Moy & ET & Moy & ET & Moy & ET & Moy & ET \\
\hline AGS & 46,34 & 7,50 & 48,70 & 4,90 & 44,94 & 2,53 & 47,58 & 6,16 & 48,03 & 5,20 & 48,40 & 5,30 & 48,63 & 3,96 & 45,52 & 3,85 \\
\hline AGMI & 38,70 & 4,94 & 37,27 & 4,00 & 38,58 & 2,60 & 37,05 & 4,19 & 36,89 & 3,80 & 37,01 & 3,77 & 37,58 & 3,66 & 39,66 & 3,67 \\
\hline AGPI & 13,64 & 3,91 & 12,76 & 2,76 & 15,17 & 4,94 & 14,09 & 3,93 & 13,77 & 4,09 & 13,32 & 3,70 & 12,46 & 2,51 & 13,51 & 2,33 \\
\hline AGPI (n-6) & 12,40 & 3,86 & 11,61 & 2,73 & 13,86 & 4,81 & 12,73 & 3,81 & 12,46 & 3,97 & 12,01 & 3,56 & 11,35 & 2,39 & 12,15 & 2,15 \\
\hline $18: 2(n-6)$ & 11,51 & 3,65 & 10,52 & 2,54 & 12,71 & 4,94 & 11,57 & 3,72 & 11,33 & 3,79 & 10,96 & 3,43 & 10,39 & 2,32 & 11,17 & 2,07 \\
\hline $20: 4(n-6)$ & 0,36 & 0,10 & 0,39 & 0,12 & 0,43 & 0,12 & 0,45 & 0,12 & 0,40 & 0,08 & 0,40 & 0,09 & 0,36 & 0,09 & 0,39 & 0,08 \\
\hline AGPI (n-3) & 1,24 & 0,50 & 1,15 & 0,41 & 1,31 & 0,31 & 1,35 & 0,39 & 1,31 & 0,30 & 1,31 & 0,54 & 1,11 & 0,34 & 1,36 & 0,55 \\
\hline $18: 3(n-3)$ & 0,88 & 0,47 & 0,72 & 0,29 & 0,87 & 0,23 & 0,87 & 0,30 & 0,79 & 0,25 & 0,84 & 0,50 & 0,72 & 0,33 & 0,95 & 0,48 \\
\hline $20: 5(n-3)$ & 0,05 & 0,02 & 0,06 & 0,03 & 0,05 & 0,01 & 0,07 & 0,04 & 0,09 & 0,04 & 0,08 & 0,05 & 0,06 & 0,04 & 0,06 & 0,03 \\
\hline $22: 5(n-3)$ & 0,12 & 0,03 & 0,13 & 0,04 & 0,14 & 0,05 & 0,13 & 0,04 & 0,15 & 0,04 & 0,15 & 0,06 & 0,11 & 0,03 & 0,12 & 0,03 \\
\hline $22: 6(n-3)$ & 0,19 & 0,05 & 0,24 & 0,11 & 0,25 & 0,13 & 0,28 & 0,13 & 0,29 & 0,10 & 0,25 & 0,12 & 0,21 & 0,10 & 0,23 & 0,11 \\
\hline AGT & 1,32 & 0,45 & 1,27 & 0,30 & 1,30 & 0,34 & 1,28 & 0,37 & 1,31 & 0,32 & 1,27 & 0,40 & 1,32 & 0,41 & 1,31 & 0,28 \\
\hline ratio LA/ALA & 13,1 & & 14,6 & & 14,6 & & 13,3 & & 14,3 & & 13,0 & & 14,4 & & 11,8 & \\
\hline
\end{tabular}

ACS : acides gras saturés; AGMI : acides gras mono-insaturés; AGPI : acides gras polyinsaturés; AGT : acides gras trans ;

$L A$ : acide linoléique ; ALA : acide alphalinolénique.

paré les résultats obtenus lors de cette étude (2007) à ceux rapportés précédemment pour la France (1993-1998). Le tableau 2 rassemble les données de six études (10-15) qui ont concerné différentes régions. On note que les compositions en AGPI n-6 et n-3 étaient relativement homogènes entre études. Les proportions d'acide linoléique variaient de 11,8\% à $15,3 \%$, avec une moyenne égale à $13,5 \%$ $\pm 1,3 \%$, celles d'acide alphalinolénique se situaient entre $0,52 \%$ et $0,70 \%$ avec une moyenne égale à $0,57 \pm 0,08 \%$. Le contenu moyen en ARA était égal à 0,39 $\pm 0,09 \%$; la somme EPA+DHA atteignait en moyenne 0,23 $\pm 0,08 \%$ des acides gras totaux. La comparaison des valeurs moyennes des 145 échantillons de l'étude présente (2007) à celles des 102 échantillons collectés dans les six études précitées (1993-1998) indique une évolution dans le temps pour les acides gras suivants : 1) l'acide linoléique a diminué de $17 \%$, passant de $13,5 \%$ à $11,1 \%$ des acides gras totaux ; 2) l'acide alphalinolénique a augmenté de $45 \%$, passant de $0,57 \%$ à $0,83 \%$. Ainsi, le rapport
LA/ALA qui était égal à 24 (1993-1998) est passé à 13,5 en 2007. Ces observations témoignent d'une évolution favorable dans le rééquilibrage des apports précurseurs oméga- 6 et oméga-3. Les contenus en ARA et DHA n'ont pas évolué significativement au cours de la période considérée.

À titre de comparaison avec d'autres pays, l'étude récente de Yuhas et al. [19] qui décrit la composition en acides gras du lait humain mature de 9 pays (Australie, Canada, Chili, Chine, Japon, Mexique, Philippines, Royaume Uni et États-Unis) indique que les proportions $\mathrm{d}$ 'acide linoléique varient du simple au double, soit 7,9\% aux Philippines contre $16 \%$ au Mexique et $18 \%$ au Chili. En France, en 2007, les proportions (11,1\% LA) sont les mêmes qu'au Royaume Uni, au Canada et en Australie (10,5-11,5 \%). Dans l'étude de Yukas, on observe également de grandes différences entre pays sur le contenu en acide alphalinolénique du lait maternel; celui-ci passe de $0,43 \%$ aux Philippines à 2,02 \% en Chine. Le rapport LA/ALA est très différent ; il est égal

Tableau 2. Composition (\% des acides gras totaux) en AGPI n-6 et n-3 du lait maternel (données françaises 19931998)

\begin{tabular}{|llllll|}
\hline & LA & ALA & ARA & EPA+DHA & LA/ALA \\
\hline Guesnet [10] & 13 & 0,62 & 0,45 & 0,37 & 21,0 \\
Martin [11] & 12,7 & 0,59 & 0,36 & 0,24 & 21,5 \\
Chirouze [12] & 15,3 & 0,46 & 0,42 & 0,41 & 33,3 \\
Chardigny [13] & 14,7 & 0,70 & 0,50 & 0,32 & 21,0 \\
Billeaud [14] & 13,32 & 0,52 & 0,38 & 0,26 & 25,6 \\
Maurage [15] & 11,8 & 0,55 & 0,24 & 0,14 & 21,5 \\
Moyenne & 13,47 & 0,57 & 0,39 & 0,23 & 24 \\
Écart type & 1,30 & 0,08 & 0,09 & 0,08 & 5 \\
\hline
\end{tabular}

à 7,6 en Chine contre 19,5 aux Philippines. Cette large étude confirme une fois de plus I'incidence de l'alimentation maternelle sur le profil en acides gras du lait humain, plus particulièrement en AGPI, les proportions d'AGS et AGMI étant quant à elles relativement constantes entre pays. L'ensemble des données conforte la connaissance sur l'origine (multiple) des acides gras présents dans le lait maternel, à savoir qu'ils proviennent d'une part de l'alimentation maternelle, soit directement, soit après avoir été stockés dans le tissu adipeux, d'autre part de la synthèse par la glande mammaire. Cette dernière concerne les AGS et la plupart des AGMI. Les AGPI n-6 et n-3, quant à eux, sont apportés par l'alimentation maternelle ; en effet, les précurseurs (LA et ALA) ne sont pas synthétisables par les cellules animales et les AGPI-LC sont préférentiellement captés préformés par la glande mammaire [11, 20]. L'objectif de notre étude était également d'apprécier l'évolution de la consommation $\mathrm{d}^{\prime} \mathrm{AGT}$ via l'évolution du contenu en AGT du lait humain au cours des dix dernières années. Pour cela, nous avons comparé les données $(n=20)$ issues du lactarium de Bordeaux (2007) à celles $(n=16)$ de l'étude Aquitaine de 1997 [21] pour laquelle nous disposions des données sur les AGT. Le tableau 3 permet de comparer les valeurs moyennes issues de ces deux études. On constate que la somme des AGT $(16: 1 t+18: 1 t+18: 2 t)$ a diminué en 10 ans de $37 \%$ passant de 2,1\% à 1,3\% des acides gras totaux du lait maternel. Compte tenu de la diminution des teneurs en AGT observée dans les margarines et dans de nombreux produits transformés au cours de cette période, cette évolution était attendue; elle se trouve ici confirmée. 
Tableau 3. Évolution de la composition (\% des acides gras totaux) en AGPI $n-6$ et $n-3$, et en acides gras trans $d u$ lait maternel entre 1997 (réf 21) et 2007 (lactarium de Bordeaux).

\begin{tabular}{|llllll|}
\hline & $\begin{array}{l}\mathbf{1 9 9 7} \\
\mathbf{n = 1 6}\end{array}$ & & \multicolumn{2}{c}{$\begin{array}{l}\mathbf{2 0 0 7} \\
\mathbf{n = 2 0}\end{array}$} & \multirow{2}{*}{ Test T } \\
\cline { 2 - 3 } & Moyenne & $\mathbf{E T}$ & Moyenne & ET & \\
\hline LA & 13,32 & 4,29 & 10,39 & 2,32 & $\mathrm{p}<0,05$ \\
ALA & 0,52 & 0,14 & 0,72 & 0,33 & $\mathrm{p}<0,05$ \\
ARA & 0,38 & 0,07 & 0,36 & 0,09 & $\mathrm{NS}$ \\
DHA & 0,26 & 0,11 & 0,21 & 0,10 & NS \\
AGT & 2,1 & 0,76 & 1,32 & 0,41 & $\mathrm{p}<0,001$ \\
LA/ALA & 25,6 & & 14,4 & & \\
\hline
\end{tabular}

\section{Conclusion}

Sur la base du contenu en AGPI n-6 et n-3 du lait humain qui est un indicateur du niveau de ces acides gras dans I'alimentation maternelle, cette étude multicentrique a permis de montrer qu'il n'y a pas de différence remarquable entre les 8 régions françaises concernées. La comparaison des données de cette large étude, conduite en 2007, à celles publiées entre 1993 et 1998 indique clairement une évolution inverse des apports en acides linoléique et alphalinolénique au cours de la dernière décennie. La diminution de l'acide linoléique associée à l'augmentation de l'acide alphalinolénique se traduit par une baisse remarquable (44\%) du rapport LA/ALA dans le lait maternel. Ce constat témoigne d'une évolution favorable dans le rééquilibrage des apports précurseurs oméga- 6 et oméga- 3 de la population française. En outre, cette étude rapporte les informations les plus récentes concernant le niveau de consommation des AGT par la population française, via leur représentation dans le lait humain. On constate qu'en 2007 la teneur en AGT du lait humain a largement diminué comparée à celle mesurée en 1997, passant de 2,1 \% à 1,3\% par rapport aux acides gras totaux. Cette diminution reflète celle des apports alimentaires en AGT de la population française.

Remerciements. Les auteurs tiennent à remercier les personnes allaitantes pour leur participation à l'étude, les équipes des lactariums, en particulier Pr Jean-Charles Picaud (Lactarium de Montpellier), Pr Chantal Maurage, (Lactarium de Tours), Pr Frédérique Huet (Lactarium de Dijon), Dr Cécile Boscher (Lactarium de Nantes), DrVirginie Rigourd (Lactarium d'île-de-France), Pr Olivier Garraud (Lactarium de St-Étienne) pour leur collaboration, et Laurence Fonseca, Sandrine
Djoukitch et Émeline Montesinos pour l'analyse des échantillons. Cette étude a été réalisée avec le soutien financier de I'ONICC (Office National Interprofessionnel des Grandes Cultures) et de I'ONIDOL (Organisation Nationale Interprofessionnelle des Oléagineux).

\section{RÉFÉRENCES}

1. Jensen RG. Lipids in human milk. Lipids 1999 ; $34: 1243-71$.

2. Innis SM. Polyunsaturated fatty acids in human milk: an essential role in infant development. Advances in Experimental Medicine and Biology $2004 ; 554$ : 27-43.

3. Uauy R, Hoffman DR, Mena P, Llanos A, Birch ER. Term infant studies of DHA and ARA supplementation on neurodevelopment: results of randomized controlled trials. J Pediatr 2003 ; $143:$ S17-25.

4. Lauritzen L, Jorgensen MH, Mikkelsen TB, et al. Maternal fish oil supplementation in lactation: effect on visual acuity and n-3 fatty acid content of infant erythrocytes. Lipids 2004 ; 39 : 195-206.

5. Fleith M, Clandinin MT. Dietary PUFA for preterm and term infants : review of clinical studies. Crit Rev Food Sci Nutr 2005 ; 45 : 205-29.

6. Read WW, Lutz PG, Tashijan A. Human milk lipids. II. The influence of dietary carbohydrates and fat on the fatty acids of mature milk. A study in four ethnic groups. Am / Clin Nutr $1965 ; 17: 180-3$.

7. Koletzko B, Thiel I, Abiodun PO. The fatty acid composition of human milk in Europe and Africa. J Pediatr $1992 ; 120$ : S62-70.

8. Innis SM. Human milk: maternal dietary lipids and infant development. Proc Nutr Soc 2007 ; $66: 397-404$.
9. Combe N, Boué C. Apports alimentaires en acides linoléique et alpha-linolénique d'une population d'Aquitaine. OCL $2001 ; 8$ : 118-21.

10. Guesnet $P$, Antoine JM, Rochette De Lempdes JB, Galent A, Durand G. Polyunsaturated fatty acid composition of human milk in France: changes during the course of lactation and regional differences. Eur / Clin Nutr 1993 ; 47 : 700-10.

11. Martin JC, Bougnoux P, Fignon A, et al. Dependence of human milk essential fatty acids on adipose stores during lactation. Am / Clin Nutr $1993 ; 58: 653-9$.

12. Chirouze V, Lapillonne A, Putet G, Salle BL. Red blood cell fatty acid composition in lowbirth-weight infants fed either human milk or formula during the first months of life. Acta Paediatr $1994 ; 405: 70-7$.

13. Chardigny JM. Wolff RI, Mager E, Sébédio Jl, Martine L, Juanéda P. Trans mono- and polyunsaturated fatty acids in human milk. Eur / Clin Nutr 1995 ; 49 : 523-31.

14. Billeaud C, Bougle D, Sarda P, et al. Effects of preterm infant formula supplementation with alpha-linolenic acid with a linoleate/alphalinolenate ratio of 6 : a multicentric study. Eur J Clin Nutr 1997 ; 51 : 520-6.

15. Maurage $C$, Guesnet $P$, Pinault $M$, et al. Effect of two types of fish oil supplementation on plasma and erythrocyte phospholipids in formula-fed term infants. Biol Neonate 1998 ; 74 : 416-29.

16. AFSSA. Rapport sur « Acides gras de la famille oméga 3 et système cardiovasculaire : intérêt nutritionnel et allégations », 54 pages. 2003

17. Pascal $G$. Les acides gras trans : origine, impact santé, évolution de leur teneur dans les aliments en France au cours des dernières années. Les mises au point de I'IFN $2009 ; n^{\circ} 3$.

18. Lepage G, Roy CC. Direct transesterification of all classes of lipids in a one-step reaction. / Lipid Res $1986 ; 27$ : 114-20.

19. Yuhas R, Pramuk K, Lien EL. Human milk fatty acid composition from nine countries varies most in DHA. Lipids $2006 ; 41: 851-8$.

20. Demmelmair $H$, Baumheuer $M$, Koletzko $B$, Dokoupil K, Kratl G. Metabolism of U13Clabeled linoleic acid in lactating women. / Lipid Res 1998 ; 39 : 1389-96.

21. Combe N, Billeaud C, Mazette B, Entressangles B, Sandler B. TFAs in human milk reflect animal or vegetal TFA consumption in France. Pediatr Res 1997 ; 37 : 304A. 